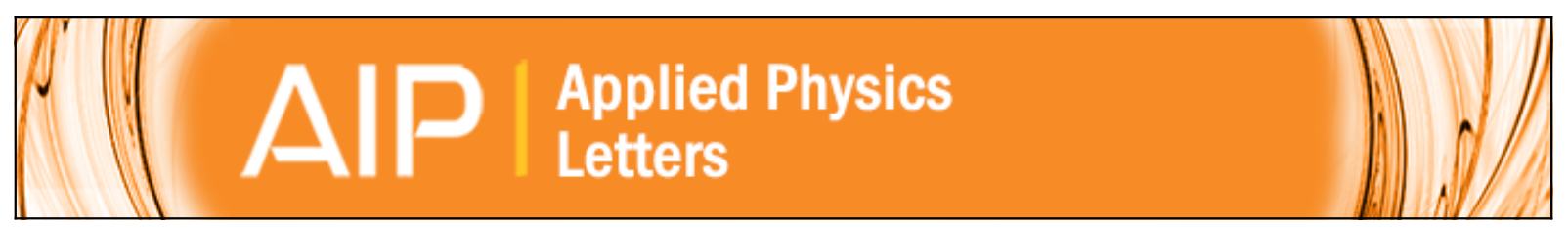

\title{
Effect of ultraviolet illumination on metal oxide resistive memory
}

José Ramón Durán Retamal, Chen-Fang Kang, Chih-Hsiang Ho, Jr-Jian Ke, Wen-Yuan Chang, and Jr-Hau He

Citation: Applied Physics Letters 105, 253111 (2014); doi: 10.1063/1.4904396

View online: http://dx.doi.org/10.1063/1.4904396

View Table of Contents: http://scitation.aip.org/content/aip/journal/apl/105/25?ver=pdfcov

Published by the AIP Publishing

\section{Articles you may be interested in}

Bipolar and unipolar resistive switching modes in $\mathrm{Pt} / \mathrm{Zn0.99Zr0.010/Pt} \mathrm{structure} \mathrm{for} \mathrm{multi-bit} \mathrm{resistance} \mathrm{random}$ access memory

Appl. Phys. Lett. 104, 183501 (2014); 10.1063/1.4875383

Stabilizing resistive switching performances of TiN/MgZnO/ZnO/Pt heterostructure memory devices by programming the proper compliance current

Appl. Phys. Lett. 104, 043508 (2014); 10.1063/1.4863744

Effects of the oxygen vacancy concentration in InGaZnO-based resistance random access memory Appl. Phys. Lett. 101, 243503 (2012); 10.1063/1.4770073

Unipolar resistive switching behavior of $\mathrm{Pt} / \mathrm{Li}$ x $\mathrm{Zn} 1-\mathrm{x}$ O/Pt resistive random access memory devices controlled by various defect types

Appl. Phys. Lett. 101, 203501 (2012); 10.1063/1.4766725

Mechanism for resistive switching in an oxide-based electrochemical metallization memory Appl. Phys. Lett. 100, 072101 (2012); 10.1063/1.3683523

\section{Model PS-100}

Tabletop Cryogenic Probe Station

\section{Lake Shore CRYOTRONICS}

An affordable solution for a wide range of research 


\title{
Effect of ultraviolet illumination on metal oxide resistive memory
}

\author{
José Ramón Durán Retamal, ${ }^{1}$ Chen-Fang Kang, ${ }^{1}$ Chih-Hsiang Ho, ${ }^{2} \mathrm{Jr}$-Jian Ke, ${ }^{1}$ \\ Wen-Yuan Chang, ${ }^{1}$ and Jr-Hau $\mathrm{He}^{1, \mathrm{a})}$ \\ ${ }^{1}$ Computer, Electrical, and Mathematical Sciences and Engineering (CEMSE) Division, King Abdullah \\ University of Science \& Technology (KAUST), Thuwal 23955-6900, Saudi Arabia \\ ${ }^{2}$ Department of Electrical and Computer Engineering, Purdue University, West Lafayette, Indiana 47907, USA
}

(Received 3 September 2014; accepted 4 December 2014; published online 24 December 2014)

\begin{abstract}
We investigate the photoelectrical and resistive switching properties of $\mathrm{Pt} / \mathrm{ZnO} / \mathrm{Pt}$ capacitor operated in unipolar mode under ultraviolet (UV) illumination. The oxygen photodesorption under UV illumination explains the photoconduction observed in initial and high resistance states. Meanwhile, oxygen readsorption at surface-related defects justifies the different photoresponses dynamics in both states. Finally, UV illumination significantly reduces the variations of resistance in high resistance state, set voltage and reset voltage by 58\%, 33\%, and 25\%, respectively, stabilizing $\mathrm{Pt} / \mathrm{ZnO} / \mathrm{Pt}$ capacitor. Our findings in improved switching uniformity via UV light give physical insight into designing resistive memory devices. (C) 2014 AIP Publishing LLC.
\end{abstract}

[http://dx.doi.org/10.1063/1.4904396]

The resistive switching (RS) properties of metal-oxidemetal capacitors have recently attracted a great deal of attention for its potential application as resistive random access memory (RRAM). ${ }^{1}$ For this reason, worldwide research groups are carrying out an unprecedented comprehensive investigation into RS properties of metal-oxide RRAM devices. $^{2}$ It is widely accepted that RS properties in oxides are based on transitions between the formation and rupture of conductive filaments (CFs) consisting of oxygen vacancies. ${ }^{3}$ However, the underlying mechanism of RS phenomena is still under debate due to the great variety of factors affecting the stability of CFs. It has been known that one of the most influential factors is the surface depletion region (SDR) caused by chemisorbed oxygen adatoms $\left(\mathrm{O}_{2(\mathrm{~g})}+e^{-} \rightarrow \mathrm{O}_{2}^{-}(\mathrm{ad})\right)$ at surface-related defects, the so-called surface effect. ${ }^{4}$ Indeed, recent studies investigating the influence of ambiences conditions, ${ }^{5}$ harsh environments, ${ }^{6}$ and roughness ${ }^{7}$ on metal oxidebased RRAM have highlighted SDR as the main cause of low switching yield and resistance fluctuation. On the other hand, the treatments such as surface modification, ${ }^{6}$ doping, ${ }^{8}$ and electrode material engineering 9 have improved RS stability and RRAM performance due to the better controllability of chemisorbed oxygen at surface-related defects. In this regard, the light illumination with the energy higher than the bandgap of metal oxide memory, which modulates the chemisorbed oxygen at surface-related defects via oxygen photodesorption $\left(\mathrm{O}_{2}^{-}(\mathrm{ad})+h^{+} \rightarrow \mathrm{O}_{2(\mathrm{~g})}\right),{ }^{10}$ is expected to have impacts on RRAM stability and performance. For example, $\mathrm{ZnO}$ with a bandgap of $\sim 3.4 \mathrm{eV}$ is sensitive to UV illumination, exhibiting high photogain upon UV illumination. ${ }^{11}$ Therefore, the implications of UV illumination during the RS of ZnO-based RRAM require further study.

In this work, the photo-electrical and RS properties of the $\mathrm{Pt} / \mathrm{ZnO} / \mathrm{Pt}$ capacitor operated in unipolar resistive switching (URS) mode under UV illumination are examined. Time-resolved photocurrent measurements under UV

\footnotetext{
${ }^{\text {a) }}$ Author to whom correspondence should be addressed. Electronic mail: jrhau.he@kaust.edu.sa
}

illumination reveal different photoresponse dynamics in the initial resistance state (IRS) and high resistance state (HRS), but not obvious photoresponse is observed in the low resistance state (LRS). A bi-exponential model is used to fit the photoresponse for gaining insight in RS behaviors and surface conditions in different resistance states. Additionally, the reliability of RRAM under UV illumination is evaluated by endurance tests, showing that resistance and switching voltage distributions are improved by UV illumination. Finally, based on the observation of the RS behaviors under UV illumination, a probable RS mechanism is proposed.

A two-terminal device consisting of a $\operatorname{Pt}(100 \mathrm{~nm}) /$ $\mathrm{ZnO}(100 \mathrm{~nm}) / \mathrm{Pt}(100 \mathrm{~nm})$ capacitor was fabricated as reported previously. ${ }^{12}$ The $\mathrm{ZnO}$ layer was deposited on a $\mathrm{Pt} /$ $\mathrm{Ti} / \mathrm{SiO}_{2} / \mathrm{Si}$ substrate by $\mathrm{RF}$ magnetron sputtering, and the $\mathrm{Pt}$ top electrode (TE) with a diameter of $200 \mu \mathrm{m}$ was deposited by DC magnetron sputtering using a shadow mask. The electrical characteristics were examined using a Keithley 4200 semiconductor parameter analyzer in dc sweep mode, air and room temperature. The UV excitation source was a SP CM110 monochromator with an average intensity of 0.31 $\mathrm{mW} / \mathrm{cm}^{2}$ at $\lambda=365 \mathrm{~nm}$. During all the measurements, the bottom electrode (BE) was grounded.

The resistance of fabricated $\mathrm{Pt} / \mathrm{ZnO} / \mathrm{Pt}$ capacitor in IRS is up to several tens of $\mathrm{M} \Omega$. An electrical stress with a current compliance $\left(I_{\text {comp }}\right)$ of $5 \mathrm{~mA}$, known as the electroforming process, is needed to initiate the switching behavior of the $\mathrm{Pt} / \mathrm{ZnO} / \mathrm{Pt}$ capacitor. As shown in Fig. 1(a), the current exhibits an abrupt increase at the voltage of $2.6 \mathrm{~V}$ (forming voltage), leading to the RS from IRS to LRS. Note that, after the electroforming process, $\mathrm{Pt} / \mathrm{ZnO} / \mathrm{Pt}$ cells have been operated in URS mode. A reset process (LRS to HRS) is carried out by sweeping voltage again without fixing the $I_{\text {comp }}$. As observed in Fig. 1(a), the current suddenly drops at the voltage of $\sim 1.1 \mathrm{~V}$ (V-Reset), giving rise to the RS from LRS to HRS. While sweeping again, an abrupt jump of current appears at a higher voltage of $\sim 2.2 \mathrm{~V}$ (V-Set); the cell returns to LRS. This bi-stable RS behavior is reversible and controllable. In this work, the CF model is adapted to explain 


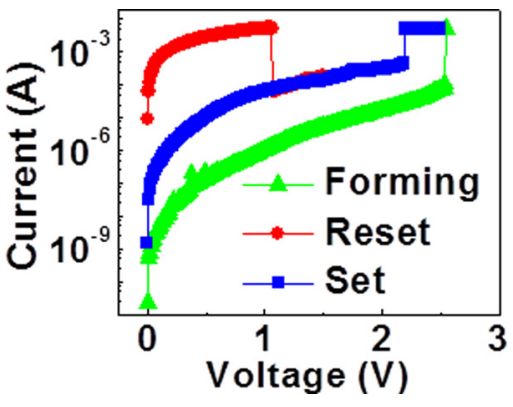

FIG. 1. The electroforming, reset, and set electrical switching cycles of the URS operation mode.

the URS behavior. CFs are formed by the alignment of structural defects, such as oxygen vacancies, which could preexist or be induced under an electric field. ${ }^{13}$ Since oxygen vacancy is positively charged in nature, it drifts toward the cathode after generation. Once the oxygen vacancies reach cathode, the nucleation of CF begins. ${ }^{3}$ With the increase of electric field, $\mathrm{CF}$ grows toward the anode. Once a CF contacts the anode, the resistance of $\mathrm{Pt} / \mathrm{ZnO} / \mathrm{Pt}$ capacitor degrades abruptly, leading to the formation of LRS. A reset process is necessary to disrupt the CF and thus induce HRS. In URS mode, the reset process is triggered by local Joule heating in the $\mathrm{CF}$ bottleneck which enhances oxygen ion migration and thus leads to a thermal dissolution of the CF via oxygen vacancy annihilation.

At this point, it is noteworthy that the oxygen gas evolution in the anodic interface forms an oxygen-rich interlayer that is able to cause oxygen chemisorption at surface defects, oxygen diffusion toward TE/air interface, void formation, ${ }^{3}$ physical deformations, and even explosions. Hence, the oxygen evolution is likely the most influential factor on the instabilities of oxides-based RRAM devices, since surfacerelated defects and oxygen vacancies compete simultaneously for the oxygen evolution. It is known that chemisorbed oxygen forms a SDR with a pronounced surface band bending $(1.53 \mathrm{eV} \text { in } \mathrm{ZnO} \text {, for example })^{14}$ that affects significantly the generation/annihilation of $\mathrm{V}_{\mathrm{O}}$ near $\mathrm{Pt}(\mathrm{TE}) / \mathrm{ZnO}$ interface and randomizes the formation/rupture of $\mathrm{CF}^{5}{ }^{5}$ To deal with this issue, we propose oxygen photodesorption via UV illumination to facilitate and stabilize the RS process.

To gain insight into the oxygen evolution at the anodic interface during the RS process, we performed time-resolved photocurrent measurements at different resistance states. As shown in Figs. 2(a)-2(c), the currents in IRS an HRS upon UV illumination increase approximately from $\sim 3.5$ to $6.5 \mu \mathrm{A}$ and from 73.5 to $77 \mu \mathrm{A}$, respectively, revealing photocurrents of 3-3.5 $\mu \mathrm{A}$. The photocurrent is ascribed to the suppression of SDR via oxygen photodesorption of chemisorbed oxygen. ${ }^{4}$ Under UV illumination with the energy higher than the $\mathrm{ZnO}$ bandgap, the photo-generated electron-hole pairs are spatially separated in $\mathrm{ZnO}$ due to the discharge of chemisorbed oxygen by photo-excited holes $\left(O_{2}^{-}(a d)+h^{+} \rightarrow O_{2(g)}\right)$. Consequently, the unpaired photo-excited electrons with increased lifetime account for the photocurrent. Meanwhile, LRS exhibits current fluctuations in both (UV on and UV off) regimes without perceptible photocurrent, suggesting the metallic nature of the CFs. A schematic of the evolution of $\mathrm{CF}$ at different resistance states is depicted in Fig. 3 to illustrate the

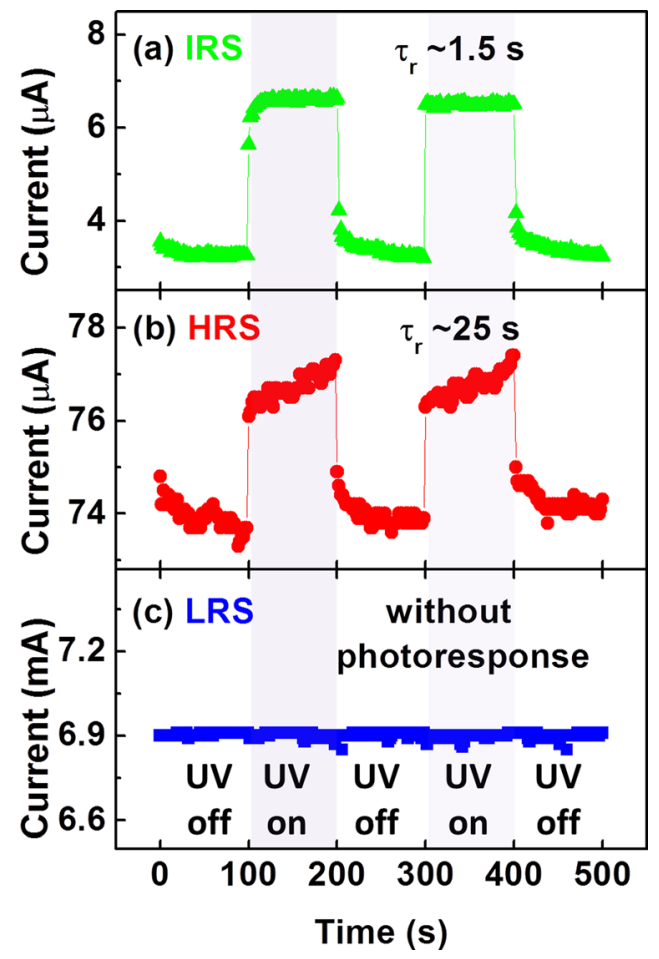

FIG. 2. Time-resolved photocurrent under $0.5 \mathrm{~V}$ bias of $\mathrm{Pt} / \mathrm{ZnO} / \mathrm{Pt}$ capacitors at UV intensity $0.31 \mathrm{~mW} / \mathrm{cm}^{2}$ in the (a) IRS (triangle), (b) HRS (circle), and (c) LRS (square).

origin of photocurrent, where the suppression of SDR under UV illumination is ascribed as the main cause responsible of photocurrent.

One can see that the photoresponse dynamics at IRS and HRS are distinct, shown in Figs. 2(a) and 2(b), respectively. Although both states exhibit a fast onset upon UV illumination exposure, the current in IRS saturates fast while grows with time in HRS. After switching off the UV illumination exposure, both states decay fast and then relax slowly toward the dark level. In order to confirm the transient dynamics, we analyze the rise time constants for IRS and HRS. For this purpose, the photoresponse can be fitted by a bi-exponential model: $I=I_{0}+A_{1} \times e^{(t / \tau 1)}+A_{2} \times e^{(t / \tau 2)}$, where $I_{0}$ represents the dark current after the photorelaxation process; $\tau_{1}$ and $\tau_{2}$

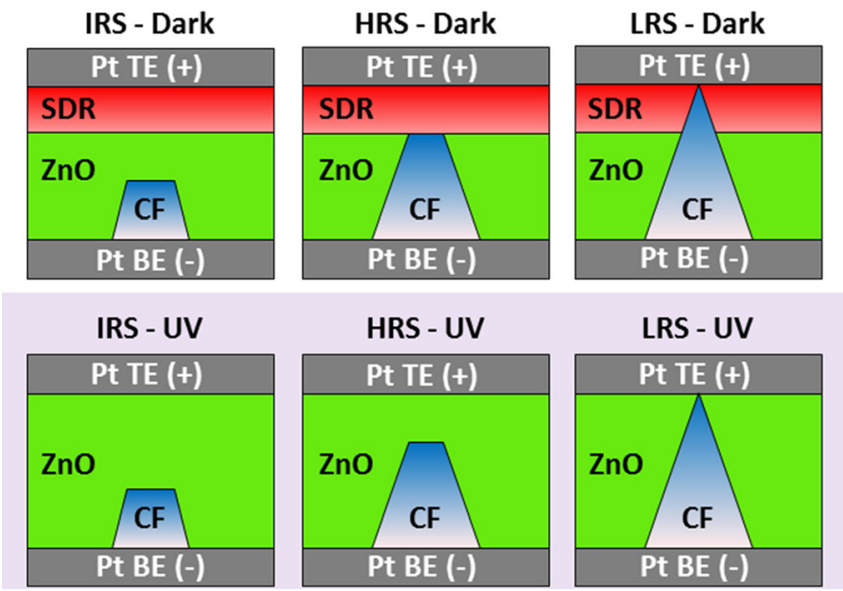

FIG. 3. Schematic of CF at IRS, HRS, and LRS under dark and under UV illumination. 

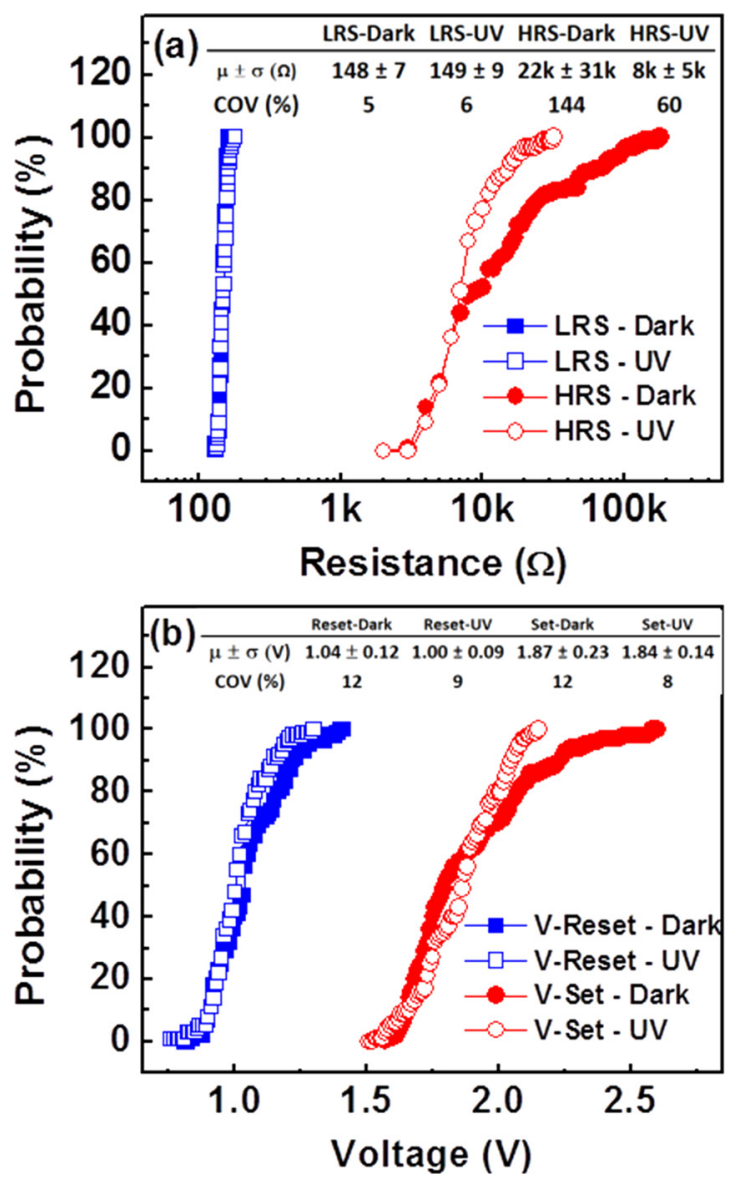

FIG. 4. The switching parameters distributions of $\mathrm{Pt} / \mathrm{ZnO} / \mathrm{Pt}$ capacitor in dark (empty symbols) and under UV illumination (solid symbols) during 100 cycling test. (a) Resistance distributions of HRS (square) and LRS (circle). (b) Switching voltage distributions of V-Set (square) and V-Reset (circle).

are the time constants, and $A_{1}$ and $A_{2}$ are weighing factors that quantify the relative contribution of each mechanism. ${ }^{15,16}$ By choosing, $\tau_{1}<\tau_{2}, \tau_{1}\left(\tau_{2}\right)$ is the shorter (longer) time constant corresponding to the faster (slower) process, which is ascribed to a bulk (surface) dominated process. Therefore, $1 / \tau_{2}$ and $1 / \tau_{1}$ are related to the surface oxygen readsorption/photodesorption rate and the bulk recombination rate, respectively. The rise time constants and relative weighted factors $\left(\tau_{1}, \tau_{2}, A_{1}\right.$, and $\left.A_{2}\right)$ for IRS are $0.01 \mathrm{~s}$, $70.15 \mathrm{~s}, 98 \%$, and $2 \%$, respectively, while for HRS are $0.41 \mathrm{~s}, 86.63 \mathrm{~s}, 71 \%$, and $29 \%$, respectively. After extracting the four parameters, we can calculate the weighted rise time constants, $\tau_{r}=A_{1}(\%) \times \tau_{1}+A_{2}(\%) \times \tau_{2} .{ }^{17}$ The calculated time constants are $\tau_{r} \sim 1.5 \mathrm{~s}$ for the IRS, and $\tau_{r} \sim 25 \mathrm{~s}$ for the HRS, confirming the slower rise in the HRS. The fast saturation in the IRS indicates the less readsorption/photodesorption sites for chemisorbed oxygen on the virgin surface/ interface of $\mathrm{Pt}(\mathrm{TE}) / \mathrm{ZnO} .{ }^{10} \mathrm{By}$ contrast, a more defective anodic $\mathrm{Pt}(\mathrm{TE}) / \mathrm{ZnO}$ interface in the HRS corresponding to a continuous readsorption/photodesorption process of chemisorbed oxygen leads to a pronounced rise behavior. ${ }^{18}$

To further explore the effects of UV illumination on RRAM characteristics, we performed endurance tests of $\mathrm{Pt} / \mathrm{ZnO} / \mathrm{Pt}$ capacitor in dark and under UV illumination. Fig. 4(a) shows the resistance distribution in terms of the cumulative probability of HRS and LRS during 100 switching cycles in the dark and under UV illumination. The resistance states were repetitively measured at read voltage $=0.1 \mathrm{~V}$. The resistance distributions of LRS in the dark and under UV illumination show a narrow distribution, indicating a high degree of stability; the UV illumination does not show any substantial effect on the distributions of the LRS, which is in agreement with our observations in the time-resolved photocurrent measurements. On the other hand, the values of HRS exhibit a highly dispersive resistance distribution under both conditions. The dispersion is a direct consequence of the random nature of the RS phenomena. Also, it is clear that, under UV illumination, the mean $(\mu)$, standard deviation $(\sigma)$, and coefficient of variation $(\mathrm{COV}=\sigma / \mu)$ of the HRS distribution are reduced by $62 \%, 84 \%$, and $58 \%$, indicating lower values and more uniform HRS distribution. Under UV illumination, the oxygen photodesorption reduces the chemisorbed oxygen concentration at the surface-related defects, suppressing the SDR, and thus reducing the resistivity to the lower values with a narrow distribution. The result is in agreement with our previous studies, ${ }^{5}$ in which the large concentration of oxygen adatoms due to external oxygen pressure results in a higher resistance in the HRS.

In addition, the switching voltage distributions of both $\mathrm{V}$-Set and V-Reset extracted from the endurance test are shown in Fig. 4(b). Under UV illumination, $\mu, \sigma$, and COV of the V-Set (V-Reset) distribution are reduced by $2 \%(4 \%)$, $39 \%(25 \%)$, and $33 \%(25 \%)$, respectively. Therefore, it is clear that UV exposure not only stabilizes the resistance distribution but also the switching voltage distribution of RRAM. It is worth to note that UV illumination has larger impact on resistance than that on voltage. According with the CF model, one of the factors to improve the uniformity of V-Set and V-Reset distributions is the stabilization of oxygen migrations. ${ }^{19}$ Thus, it is possible that by irradiating the $\mathrm{Pt} / \mathrm{ZnO} / \mathrm{Pt}$ capacitor with UV illumination, the oxygen photodesorption may help stabilize the oxygen migration and could also confine the CFs, thus minimizing the dispersion of switching voltages.

In this work, we investigated photoelectrical and RS properties of $\mathrm{Pt} / \mathrm{ZnO} / \mathrm{Pt}$ capacitor operated in URS mode and under UV illumination. Both IRS and HRS exhibit photoresponse due to oxygen photodesorption while LRS is insensitive to UV light due to the metallic nature of CFs. In addition, we observe distinct photoresponse at IRS and HRS due to different oxygen readsorption dynamics at the surfaces with different degree of surface-related defect density. On the other hand, the stabilization of the RRAM performance under UV illumination suggests that oxygen photodesorption at the $\mathrm{Pt} / \mathrm{ZnO}$ anodic interface effectively suppresses the SDR, improving the uniformity of HRS distribution, and stabilizes the oxygen migration minimizing the dispersion of V-Set and V-Reset distributions. Overall, this investigation highlights the influence of UV illumination on RS properties of oxide-based RRAM devices, paving a way for deeper understanding of RS phenomena via oxygen modulation at surfaces.

${ }^{1}$ R. Waser, R. Dittmann, G. Staikov, and K. Szot, Adv. Mater. 21, 2632 (2009).

${ }^{2}$ H. S. P. Wong, H. Y. Lee, S. Yu, Y. S. Chen, Y. Wu, P. S. Chen, B. Lee, F. T. Chen, and M. J. Tsai, Proc. IEEE 100, 1951 (2012). 
${ }^{3}$ K. M. Kim, D. S. Jeong, and C. S. Hwang, Nanotechnology 22, 254002 (2011).

${ }^{4}$ C. Y. Chen, M. W. Chen, J. J. Ke, C. A. Lin, J. R. Durán Retamal, and J. H. He, Pure Appl. Chem. 82, 2055 (2010).

${ }^{5}$ J. J. Ke, Z. J. Liu, C. F. Kang, S. J. Lin, and J. H. He, Appl. Phys. Lett. 99, $192106(2011)$

${ }^{6}$ T. H. Huang, P. K. Yang, D. H. Lien, C. F. Kang, M. L. Tsai, Y. L. Chueh, and J. H. He, Sci. Rep. 4, 4402 (2014).

${ }^{7}$ Z. Ji, Q. Mao, and W. Ke, Solid State Commun. 150, 1919 (2010).

${ }^{8}$ T. H. Huang, P. K. Yang, W. Y. Chang, J. F. Chien, C. F. Kang, M. J. Chen, and J. H. He, J. Mater. Chem. C. 1, 7593 (2013).

${ }^{9}$ P. K. Yang, W. Y. Chang, P. Y. Teng, S. F. Jeng, S. J. Lin, P. W. Chiuh, and J. H. He, Proc. IEEE 101, 1732 (2013).

${ }^{10}$ D. H. Zhang, Mater. Chem. Phys. 45, 248 (1996).

${ }^{11}$ J. R. D. Retamal, C. Y. Chen, K. Y. Lai, and J. H. He, Handbook of Zinc Oxide and Related Materials (Taylor \& Francis, 2012), Vol. 2, Chap. IV, pp. 133-174.
${ }^{12}$ W. Y. Chang, Y. C. Lai, T. B. Wu, S. F. Wang, F. Chen, and M. J. Tsai, Appl. Phys. Lett. 92, 022110-1 (2008).

${ }^{13}$ J. J. Yang, F. Miao, M. D. Pickett, D. A. A. Ohlberg, D. R. Stewart, C. N. Lau, and R. S. Williams, Nanotechnology 20, 215201-1 (2009).

${ }^{14}$ C. Y. Chen, J. R. D. Retamal, I. W. Wu, D. H. Lien, M. W. Chen, Y. Ding, Y. L. Chueh, C. I. Wu, and J. H. He, ACS Nano 6, 9366 (2012).

${ }^{15}$ C. Y. Chen, M. W. Chen, C. Y. Hsu, D. H. Lien, M. J. Chen, and J. H. He, IEEE J. Sel. Top. Quantum Electron. 18, 1807 (2012).

${ }^{16}$ J. R. D. Retamal, C. Y. Chen, D. H. Lien, M. R. S. Huang, C. A. Lin, C. P. Liu, and J. H. He, ACS Photonics 1, 354 (2014).

${ }^{17}$ P. R. Barber, S. M. Ameer-Beg, J. Gilbey, L. M. Carlin, M. Keppler, T. C. $\mathrm{Ng}$, and B. Vojnovic, J. R. Soc. Interface 6, S93 (2009).

${ }^{18}$ L. Luo, B. D. Sosnowchik, and L. Lin, Nanotechnology 21, 495502 (2010).

${ }^{19}$ V. Y. Q. Zhuo, Y. Jiang, R. Zhao, L. P. Shi, Y. Yang, T. C. Chong, and J. Roberton, IEEE Electron Device Lett. 34, 1130 (2013). 